\title{
Rothia dentocariosa
}

National Cancer Institute

\section{Source}

National Cancer Institute. Rothia dentocariosa. NCI Thesaurus. Code C86728.

A species of aerobic, Gram positive, rod shaped bacteria assigned to the phylum

Actinobacteria. This species cannot oxidize glucose or hydrolyze starch but is able to degrade gelatin and reduce nitrite. $R$. dentocariosa is a part of the normal flora of the mouth, nose and throat and can cause subacute infective endocarditis with extracardiac complications in immunocompromised patients. 